\title{
Experimental study on allografts of amniotic epithelial cells in calcaneal tendon lesions of sheep
}

\author{
A. Muttini • M. Mattioli • L. Petrizzi • V. Varasano • \\ C. Sciarrini • V. Russo • A. Mauro • D. Cocciolone • \\ M. Turriani • B. Barboni
}

Published online: 22 May 2010

(C) Springer Science+Business Media B.V. 2010

\begin{abstract}
An experimental protocol was designed to study the survival and behaviour of an allograft of amniotic epithelial cells (AECs) in an ovine model. The study was conducted on three healthy adult sheep. A core lesion was created in both calcaneal tendons under ultrasound (US) guidance by injecting $400 \mathrm{UI}$ of Type 1A collagenase diluted in $0.6 \mathrm{ml}$ saline. The AECs were obtained from a 60-80-day-old fetus and cultured under standard conditions. After 15 days of collagenase treatment, $2 \times 10^{6}$ AECs stained with a vital membrane fluorescent probe (PHK26) were injected under US guidance in $500 \mu$ l saline solution into the lesion of one limb. The contralateral untreated limb was used as a control. Animals were euthanatized 7 (1) and 30 (2) days later. Histological analyses performed on explanted tendons clearly demonstrate that AECs survived for at least 1 month inside the lesion without any adverse reactions. The damaged tissue of the treated tendons showed a high number of reparative cells in active proliferation that were accumulating collagen within the extracellular matrix. In addition, after 1 month, the neo-collagen began to be organized into parallel arrays of fibers oriented along the longitudinal axis of the tendon.
\end{abstract}

Keywords Allograft · Amniotic epithelial cells $\cdot$ Calcaneal tendon $\cdot$ Sheep

\author{
Abbreviations \\ AECs amniotic epithelial cells \\ US Ultrasound \\ HE Herovici histological staining
}

\footnotetext{
A. Muttini $(\bowtie) \cdot$ L. Petrizzi $\cdot$ V. Varasano $\cdot$ C. Sciarrini

Department of Veterinary Clinical Sciences, University of Teramo,

Viale Crispi 212, Teramo, TE 64100, Italy

e-mail: amuttini@unite.it
}

M. Mattioli • V. Russo $\cdot$ A. Mauro - D. Cocciolone $\cdot$ M. Turriani $\cdot$ B. Barboni

Department of Biomedical Sciences, University of Teramo, Teramo, Italy 


\section{Introduction}

Ligament and tendon injuries, especially of the superficial digital flexor tendons (SDFT), are a substantial cause of wastage within the horse industry (Dowling et al. 2000). The reported incidence reaches $42 \%$ in racing thoroughbreds (Wilson et al. 1996). Low performance syndrome is sometimes present regardless of clinical signs. Additionally, in humans, tendon lesions are an important problem particularly in athletes (Abate et al. 2009). Regenerative therapy with bone marrow autografts in horses dates from 2001 (Hertel 2001). In Italy, this protocol was previously described and demonstrated good results (Muttini et al. 2006). The clinical efficacy of stem cell implantation has been reported by many experimental studies and clinical trials. However, comparative studies focused on stem cells from other sources and characterization of the precise cell type is lacking.

Recently, the distinguishing features of stem cells taken from amniotic membranes (Niknejad et al. 2008) or from amniotic fluid (Siegel et al. 2008) have been described. Briefly, the most important characteristics of these cells are the anti-inflammatory and antimicrobial properties, and low immunogenicity. Based on these features, the use of these cells in regenerative treatment of tendinopathies could be utilized in both veterinary and human medicine. This preliminary report shows the results of the allotransplantation of amniotic epithelial cells (AECs) into experimentally-induced lesions of calcaneal tendons of adult sheep. The aims of this study were 1) to verify the feasibility of the AEC allograft without inducing immunologic or other adverse reactions, 2) to demonstrate the survival of AECs in the experimental defects, and 3) to verify by histological examination of the tissue if the AECs could induce regeneration rather than scar formation.

\section{Materials and methods}

A bilateral defect was created in the middle portion $(5 \mathrm{~cm}$ proximal to the calcaneal tuberosity) of the Achilles tendon (calcaneal tendon) of three 50-kg adult sheep. The defect was realized as previously described (Crovace et al. 2008) by injecting 400 UI of collagenase 1 (Sigma tau) in $0.6 \mathrm{ml}$ saline under ultrasound (US) guidance. After 7 days the defect was detected by ultrasonographic examination in transversal and longitudinal scans. AECs were collected from the placenta of slaughtered sheep at 60-80 days of pregnancy. AECs were obtained from the epithelial layer of the amniotic membrane by enzymatic digestion (EDTA-trypsin) and cultured in standard conditions (Parolini et al. 2009) for three passages. Before grafting, AECS were stained with a vital membrane fluorescent probe (PHK26) to identify the transferred cells in the host tissue. AECs were transferred into the experimental defect under US guidance. A total of $2 \times 10^{6}$ AECs in $500 \mu$ l saline were grafted into the experimental defects 15 days after the collagenase injection. The contra lateral untreated limb was used as a control. Animals were stabled and observed daily for signs of discomfort. One sheep was euthanatized after 7 days; two animals were euthanatized after 30 days.

Tendon samples obtained in correspondence to the experimental defect were collected. The specimens were frozen in liquid nitrogen and cryosectioned at $10 \mu \mathrm{m}$. Sections were analyzed for 1) haematoxylin-eosin and Herovici (HE) histological staining, 2) nuclear contrast with fluorescent DAPI staining, and 3) immunoreaction with a cellular proliferation marker Ki-67 (Santa Cruz Biotechnology) detected by a secondary anti-mouse AlexaFluor 488-conjugated antibody. All the sections were analyzed with an Axioscope 2 microscope (Zeiss) at $\times 100$ and $\times 400$ magnification. 


\section{Results}

The experimental protocol was tolerated well by all animals. A slight bilateral lameness was noted a few days after collagenase administration. Markedly hypoechoic core lesions were detected 7 days after the injection. One lesion was clearly lateral due to a technical mistake and was used for morphological validation 7 days after the collagenase injection. Macroscopic examination revealed some inflammatory reaction in the collagenase injection site 7 days after allograft. On the contrary, inflammation was absent in 30-day explanted tendons. Histological examination revealed damaged tissue 7 days post-grafting. The extracellular matrix appeared to be composed of a few collagen fibers with an irregular distribution as demonstrated by HE. A high number of cells was identified in the damaged tendon. No proliferative (Ki67 negative) PKH26 positive AECs were observed within the lesion site. The control tendon showed an altered organization similar to that observed in the allografted tendon even with a minor cellularity. The presence of inflammatory cells was similar in the grafted and control tendon. Tendon sections obtained from the animals sacrificed after 30 days, displayed AECs distally and marginally to the defect. Many proliferating cells (Ki67-positive) were identified near AECs, some of which had flat nuclei and were enclosed within collagen fibers that started to be oriented along the longitudinal tendon axis (HE). In control specimens, no tendon healing was observed 30 days after collagenase injection. In fact, histological examination revealed no proliferating cells with little non-organized collagen deposition.

\section{Discussion}

The reported data supported the experimental hypothesis. The AEC allograft did not induce any immunological reaction in the host tissue up to one month after implant. This data confirms the low immunogenicity of amniotic-derived cells (Niknejad et al. 2008). The inflammation clinically noted subsided in a few days. Histological examination demonstrated the survival of labeled AECs in the experimental lesions until 1 month after grafting. A relevant production of newly formed collagen fibers followed stem cell allotransplantation that, one month later, started to assume a parallel orientation along the axis of the tendon. The presence of several proliferating cells near inactive AECs, localized between healthy and damaged tissue, led to the hypothesis that transferred stem cells could not participate directly in the regenerative process. Their role in the regenerative process, as opposed to their role in other tissues, could be explained with the production of growth/chemotactic factors able to activate tendon regeneration. These preliminary data need to be experimentally confirmed using increased numbers of animals and by using specific markers to detect the type of collagen deposited. Long-term follow-up is also required to confirm the absence of immunogenicity.

\section{References}

Abate M, Gravare-Silbernager K, Siljeholm C, Di Iorio A, De Amicis D, SaliniV, Werner S, Paganelli R (2009) Pathogenesis of tendinopathies: inflammation or degeneration? Arthritis Res Therap 11:235-250

Crovace A, Lacitignola L, Francioso E, Rossi G (2008) Histology and immunohistochemestry study of ovine tendon grafted with cBMSC and BMMNCs after collagenase-induced tendonitis. Vet Comp Orthop Traumatol 3:1-8 
Dowling BA, Dart AJ, Hodgson DR, Smith RKW (2000) Superficial digital flexor tendonitis in the horse. Equine Vet J 32:369-378

Hertel DJ (2001) Enhanced suspensory ligament healing in 100 horses by stem cells and other bone marrow component. Proc Am Assoc Equine Pract 47:319-321

Muttini A, Cuomo A, Valerii V, Spadari A (2006) Studio retrospettivo sul trapianto autologo di midollo osseo emopoietico nel trattamento delle lesioni tenodesmiche della mano e del piede del cavallo. Atti SICV.

Niknejad H, Peirovi H, Joriani M, Ahmadiani A, Ghanavi J, Seifalian AM (2008) Properties of the amniotic membrane for potential use in tissue engineering. Euro Cells Materials 15:88-99

Parolini O, Soncini M, Evangelista M, Schmidt D (2009) Amniotic membrane and amniotic fluid-derived cells: potential tools for regenerative medicine? Regen Med 4:275-291

Siegel N, Rosner M, Hanneder M, Freiliger A, Hengstschlager M (2008) Human amniotic fluid stem cells: a new perspective. Amino Acids 35:291-293

Wilson JH, Robinson RA, Jensen RC, McArdle CJ (1996) Equine soft tissue injuriesassociated with racing: Descriptive statistics from American racetracks. In: Proc Dubai Equine Intl Symp Eds: Rantanen NW, Hauser ML:1-21 